\title{
DHA supplementation improves behavioral memory impairment and alleviates oxidative stress in hippocampal caused by repeated anesthesia of sevoflurane in aged rats
}

\author{
Ming Tian \\ Qindao University Medical College Affiliated Yantai Yuhuangding Hospital \\ Kezhong Li \\ Qindao University Medical College Affiliated Yantai Yuhuangding Hospital \\ Xiaoling Zhao ( $\nabla$ xiaoling0125@yeah.net) \\ Qindao University Medical College Affiliated Yantai Yuhuangding Hospital
}

\section{Research article}

Keywords: DHA, sevoflurane, repeated anesthesia, Nrf2/HO-1 signaling pathway

Posted Date: September 30th, 2020

DOl: https://doi.org/10.21203/rs.3.rs-29872/v2

License: (c) (1) This work is licensed under a Creative Commons Attribution 4.0 International License.

Read Full License 


\section{Abstract}

Backgroud: The current study aimed to explore the effects of docosahexaenoic acid (DHA) on the behavioral memory impairment induced by repeated anesthesia of sevoflurane in aged rats.

Methods: A total of 54 Sprague-Dawley (SD) aged rats were randomly divided into five groups: Blank control group (Control), sevoflurane group (Model), DHA group ( $3 \mathrm{~g} / \mathrm{kg})$, Sev + DHA (0.3g/kg) group, Sev + DHA $(1 \mathrm{~g} / \mathrm{kg})$ group and Sev + DHA $(3 \mathrm{~g} / \mathrm{kg})$ group. Morris water maze experiment was used to evaluate the learning and memory ability. Hematoxylin and eosin (H\&E) staining was used to observe histological changes in the hippocampus. Enzyme linked immunosorbent assay (ELISA) was used to detect the content of superoxide dismutase (SOD), malondialdehyde (MDA) and glutathione peroxidase (GSH-Px) in hippocampus. Immunohistochemistry and western blot analysis were used to determine the expression of the Nuclear factor erythroid-2 (NF-E2)-related factor 2(Nrf2)/ hemeoxygenase-1 (HO-1) signaling pathway.

Results: Rats were indicated to exhibit prolonged escape latency following sevoflurane anesthesia. The number of times taken to cross the platform and the time for target quadrant stay were also demonstrated to be significantly reduced. Rats treated with different doses of DHA were revealed to exhibit reduced escape latency. The number of times taken to cross the platform and the time for target quadrant stay increased. Histopathological examination indicated that DHA ameliorated the brain function of the rats that were repeatedly anesthetized using sevoflurane. Furthermore, the expression of Nrf2 and HO-1 protein were demonstrated to be significantly increased.

Conclusions: The present study revealed that DHA has a protective effect on learning and memory impairment in aged rats induced by repeated sevoflurane anesthesia, and the mechanism may be associated with the Nrf2/HO-1 signaling pathway.

\section{Backgroud}

Sevoflurane is a volatile anesthetic that is widely used in clinical anesthesia due to the fact it exhibits high efficiency, is easy to control, exhibits less irritation to the body and has a quick induction and recovery period. However, sevoflurane has potential neurotoxicity and has been previously reported to be associated with postoperative cognitive impairment in elderly patients [1]. In a juvenile animal study, it was demonstrated that sevoflurane mediates neurologic damage and brain dysplasia through oxidative stress [2].

DHA is an unsaturated fatty acid, which is an important component of the neuronal cell membrane [3]. DHA conducts signal transduction between brain function and nerve cells to prevent cytoskeletal protein degradation and the inhibition of oxidative stress and lipid peroxidation. Ageing is associated with changes in the DHA content of brain membranes that could contribute to memory impairment [4]. An agerelated decrease in DHA content in the hippocampus has been observed [5, 6]. The current study explored the effect of different doses of DHA on behavioral memory impairment induced by repeated sevoflurane 
anesthesia in aged rats, and aimed to provide experimental data for the side effects of repeated sevoflurane anesthesia.

A previous study indicated that DHA induces activation of Nuclear factor erythroid-2-related factor 2 (Nrf2), and subsequently expression of heme oxygenase 1 (HO-1) [7]. The / Nrf2/HO-1 signaling pathway is a multi-organ protection chain that is induced under a number of different stress environments [8]. The Nrf2/HO-1 signaling pathway exerts a variety of different effects, including anti-oxidation, antiinflammatory, reduction of mitochondrial damage and regulation of cell death [9-12]. A recent study reported that Nrf2/HO-1 play a neuroprotective role in delaying the occurrence of Alzheimer's disease [12]. However, there is no clear evidence of its role in repeated anesthesia. Therefore, in the current study, a sevoflurane repeated anesthesia aged rat model was established to determine the role and mechanism of DHA in sevoflurane repeated anesthesia, as well as providing a theoretical basis for exploring the molecular mechanisms of DHA treatment.

\section{Methods}

\section{Experimental animals}

A total of 54 aged SD male rats (Ji'nan Peng Yue Experimental Animal Breeding Co., Ltd.; production license no. SCXK (LU) 20140007; age, 18 months; weight, $540 \pm 50 \mathrm{~g}$ ) were used in the current study. All animals were quarantined as required. During the quarantine period, animal activities, including diet, were observed. Animals were required to pass quarantine prior to participation in the subsequent experiment. The environmental conditions for laboratory animal feeding and management were as follows: Room temperature $20-26^{\circ} \mathrm{C}$, daily temperature difference $\leq 4^{\circ} \mathrm{C}$, relative humidity $40-70 \%$, alternation between light and dark $12 / 12 \mathrm{~h}$. During the quarantine and experimental period, the rats had access to food and water ad libitum. During housing, animals were monitored twice daily for health status. No adverse events were observed. All experimental protocols were conducted according to the Nursing and Use Guidance for Animal Experiment Operation guidelines of the National Institutes of Health (NIH Pub. no. 85-23; revised in 1996) and were approved by the Animal Protection and Use Committee of the Affiliated Yantai Yuhuangding Hospital of Qingdao University. All sections of this report adhere to the ARRIVE Guidelines for reporting animal research [13]. A completed ARRIVE guidelines checklist is included in Checklist S1.

\section{Animal grouping, anesthesia and administration}

A total of 54 sprague dawley rats were randomly divided into 6 groups: Blank control group (Control), sevoflurane (NMPN-H20070172; Shanghai Hengrui Pharmaceutical Co., Ltd.) group (Model), DHA group (Rongcheng Baihe Biotechnology Co., Ltd.; $3 \mathrm{~g} / \mathrm{kg})$, Sev + DHA (0.3g/kg) group, Sev + DHA (1 g/kg) group, Sev + DHA $(3 \mathrm{~g} / \mathrm{kg})$ group with 9 rats in each group.

Rats in the Model, Sev + DHA (0.3g/kg) group, Sev + DHA (1 g/ $/ \mathrm{kg})$ group, Sev + DHA (3g/kg) groups were placed in a self-made transparent anesthesia box $(50 \times 40 \times 40 \mathrm{~cm})$. One side hole of the box was connected 
to a Drager anesthesia machine (Drager Company, Germany), which administered the rats' with $2.5 \%$ sevoflurane anesthetic for $3 \mathrm{~h}$, meanwhile the heart rate (HR), respiratory frequency (RF) and blood oxygen saturation (BOS) were continuously monitored using an electrocardiogram monitor (NORDEP LTD) to eliminate brain damage caused by hypoxia. When the rats were induced into a coma, they were immediately exposed to air to wake them. After their righting reflex was recovered and they were able to move freely, the rats were then placed in the box again for another dose of anesthetic. The control group received no treatment.

Rats in DHA, Sev + DHA (0.3g/kg), Sev + DHA (1 g/ kg), Sev + DHA (3g/kg) groups received daily intragastric administration of DHA on days 1-10 of the experiment (10 days prior to the administration of sevoflurane), while the Control and Model group received no treatment. On days 11 - 15 rats in the Model received daily inhalation of sevoflurane for repeated anesthesia, Sev + DHA $(0.3 \mathrm{~g} / \mathrm{kg})$, Sev + DHA $(1 \mathrm{~g} / \mathrm{kg})$, Sev + DHA $(3 \mathrm{~g} / \mathrm{kg})$ groups received DHA administration and daily inhalation of sevoflurane for repeated anesthesia, DHA group received DHA administration, while the Control group received no treatment.

Rats in the Model, Sev + DHA (0.3g/kg), Sev + DHA (1 g/ kg), Sev + DHA (3g/kg) groups received sevoflurane inhalation for anesthesia or DHA administration with the Morris water maze (MWM) test was carried out from 16 - 20 days, and no drugs was administered on day 21 before the test. The body weight of rats in each group was recorded on day 1 , day 7 , day 14 and day 21 . The experimental flow chart was shown in Figure 1.

\section{Morris water maze experiment}

\section{Learning capability test (positioning navigation experiment)}

Rat navigational abilities were assessed using Morris water maze experiments. The MWM (Institute of Materia Medica; Chinese Academy of Sciences) consisted of two parts: One round pool $(120 \mathrm{~cm}$ in diameter; $50 \mathrm{~cm}$ in height; stainless steel) and one movable platform. The platform was located $2 \mathrm{~cm}$ below the water. The water temperature of the pool was $\sim 25^{\circ} \mathrm{C}$. The pools surface was divided into four parts and was set as four quadrants (quadrants 1, 2, 3 and 4). Within a specified period of time or when the rats begin to swim until they climbed onto the platform, the computer automatically tracked the rats' swimming trajectories and calculated the incubation period (the time when the rats located the platform in the pool) automatically using software (Etho Vision XT, Noldus Information Technology BV). The positioning navigation experiment recorded the time taken for the rats to locate the platform hidden under the water surface, and tested the spatial orientation learning ability of the rats.

Rats entered the swimming test from the center of any quadrant facing the pool wall. If the animal failed to locate the platform in the pool or climbed up the platform within $120 \mathrm{sec}$, then time taken was calculated as $120 \mathrm{sec}$. The rat was then guided and placed on the platform for $30 \mathrm{sec}$. The rats were subsequently removed from the platform and wiped dry. After the rats rested for $60 \mathrm{sec}$, they were trained for the next round. Rats were trained four times a day for five consecutive days. The average of the rats' four training incubation periods was taken as the rat's daily learning achievement. 


\section{Memory test (space exploration experiment)}

A period of $24 \mathrm{~h}$ after the end of the positioning navigation experiment, and on day 21 of the experiment, the platform was withdrawn and the rats were placed in water at any of the same points of entry. The point of entry was the same as the position in the positioning navigation experiment. The rats were allowed to swim for $120 \mathrm{sec}$, and the time of target quadrant stay and the number of attempts to cross the platform were recorded.

\section{H\&E staining}

After the rats' behavioral tests were completed, all animals were sacrificed by cervical spine dislocation after anesthetized by intraperitoneal injection of $1 \%$ pentobarbital sodium $40 \mathrm{mg} / \mathrm{kg}$. Brain tissues were dissected out and placed in an ice bath, with the hippocampus being isolated from the brain. Histopathological examination was then performed using formalin fixation. The samples were embedded in paraffin and cut into $4 \mu \mathrm{m}$ sections. The paraffin sections were dewaxed using xylene, dehydrated with serial dilutions of ethanol, stained with hematoxylin and eosin (H\&E) (Beijing Solarbio Science \& Technology Co., Ltd.), and rinsed in distilled water for $30 \mathrm{sec}$. The sections were washed with $95 \%$ anhydrous ethanol, soaked twice for $1 \mathrm{~min}$, placed in a xylene soak three times for $5 \mathrm{~min}$, and permount mounting medium (Thermo Fisher Scientific, Inc.) was used to place the samples on a coverslip, and pathological changes were observed using an optical microscope (magnification, $\times 100$; Olympus Corporation).

\section{Enzyme linked immunosorbent assay (ELISA)}

The hippocampal tissue was collected from each group of rats and separated via centrifugation at $12,000 \times \mathrm{g}$ for $30 \mathrm{~min}$, at $4^{\circ} \mathrm{C}$. The supernatant was collected and the contents SOD, MDA and GSH-Px were detected by ELISA kits (Nanjing Jiancheng Bioengineering Institute, Nanjing, China), according to the manufacturer's protocol.

\section{Immunohistochemistry}

The hippocampal tissues were embedded in paraffin and sectioned $(5 \mu \mathrm{m})$. The sections were deparaffinized with xylene twice, and rehydrated in a descending ethanol series. Endogenous peroxidase was inhibited by incubating the sections for 30 min using $3 \% \mathrm{H}_{2} \mathrm{O}_{2}$. Antigen retrieval was performed using a citrate buffer at a high temperature for $10 \mathrm{~min}$. The sections were subsequently blocked for 20 min in 5\% BSA, and incubated using a primary anti-HO-1 antibody (1:100; cat. no. ab13243; Abcam), and an anti-Nrf2 antibody (1:100; cat. no. ab31163; Abcam) overnight at $4^{\circ} \mathrm{C}$. After rewarming, sections were incubated with a horseradish peroxidase (HRP)-conjugated goat anti-rabbit IgG secondary antibody (1:1000; cat. no. ab6721; Abcam) at $37^{\circ} \mathrm{C}$ for $1 \mathrm{~h}$. Sections were visualized with 3, 3-diaminobenzidine tetrahydrochloride (DAB) as the chromogen (Beijing Solarbio Science \& Technology Co., Ltd.). The sections were dehydrated and placed onto a coverslip with Permount mounting medium (Thermo Fisher Scientific, Inc.). The slides were observed under an optical microscope (Olympus Corporation; 
magnification, $\times 200$ ). The data were expressed as the percentage of positive cells out of the total number of cells counted.

\section{Western blot analysis}

Total protein of the hippocampal tissues was extracted using RIPA lysis buffer containing proteinase inhibitor cocktail, and the concentration of protein was determined using a BCA assay. Proteins $(50 \mu \mathrm{g})$ were separated using 10\% SDS-PAGE and transferred onto PVDF membranes (EMD Millipore). The membranes were blocked using $5 \%$ skim milk at $4^{\circ} \mathrm{C}$ overnight, then incubated with primary anti Nrf2 antibody (1:1,000; cat. no. ab137550; Abcam) and anti H0-1 antibody (1:2,000; cat. no. ab13243, Abcam) at $4^{\circ} \mathrm{C}$ overnight. After incubating with secondary antibody sheep anti-rabbit IgG $(1: 5,000 ;$ cat. no. ab97095; Abcam) at $37^{\circ} \mathrm{C}$ for $1 \mathrm{~h}$, protein bands were visualized using the ECL chemiluminescence system (Thermo Fisher Scientific, Inc.). Protein expression levels were normalized to $\beta$-actin (1:2,000; cat. no. ab8227; Abcam) and quantified using Image J software version 1.46 (National Institutes of Health).

\section{Statistical analysis}

All experimental data are expressed as mean \pm standard deviation, and processed using SPSS 20.0 (IBM Corp.). Multiple comparisons were evaluated using a repeated measures ANOVA. A one-way analysis of variance (ANOVA) was used to compare the mean of multiple groups with a Dunnett's post-hoc test. $\mathrm{P}<0.05$ was considered to indicate a statistically significant difference.

\section{Results}

\section{Change in body weight, $\mathrm{HR}$, RF and BOS of rats}

The body weight of rats in each group increased gradually within 3 weeks, and there was no significant difference compared with Control group $(P>0.05)$. The body weight of rats in model group had a slow trend (as shown in Table 1). On days 21, the body weight of rats in model group was significantly decreased compared to Control and DHA groups $(P<0.05)$.

As shown in Table 2, the HR and BOS in Model, Sev + DHA (0.3g/kg), Sev + DHA (1 g/ kg), Sev + DHA $(3 \mathrm{~g} / \mathrm{kg})$ groups were decreased compared with Control and DHA groups. DHA treatment caused a significant increase in HR and BOS in Sev + DHA (0.3g/kg), Sev + DHA $(1 \mathrm{~g} / \mathrm{kg})$, Sev + DHA (3g/kg) groups compared with Model group $(P<0.05)$. The RF in Model, Sev + DHA $(0.3 \mathrm{~g} / \mathrm{kg})$, Sev + DHA $(1 \mathrm{~g} / \mathrm{kg})$, Sev + DHA $(3 \mathrm{~g} / \mathrm{kg})$ groups were increased compared with Control and DHA groups. There was no significant difference between Control group and DHA group.

\section{Rat learning ability test}

Compared with Control and DHA group, the escape latency of rats in the Model group was demonstrated to be significantly increased after inhalation of sevoflurane $(P<0.05)$. Compared with the Model group, the escape latency of rats in the Sev + DHA $(0.3 \mathrm{~g} / \mathrm{kg}), \operatorname{Sev}+\mathrm{DHA}(1 \mathrm{~g} / \mathrm{kg}), \mathrm{Sev}+\mathrm{DHA}(3 \mathrm{~g} / \mathrm{kg}) \mathrm{group}$ were 
decreased, exhibited a dose-dependence on DHA, and the Sev + DHA (3g/kg) group was indicated to be significantly different $(P<0.05$; Table 3$)$.

\section{Rat memory ability test situation}

As presented in Fig. 2, compared with the Control and DHA groups, the number of times to cross the platform and the time for target quadrant stay of rats in the Model group was significantly increased after inhalation of sevoflurane $(P<0.05)$. Compared with the Model group, the number of times to cross the platform and the time for target quadrant stay of rats in the Sev + DHA $(0.3 \mathrm{~g} / \mathrm{kg})$, Sev + DHA $(1 \mathrm{~g} / \mathrm{kg})$, Sev + DHA $(3 \mathrm{~g} / \mathrm{kg})$ groups were increased, and the H-DHA group was indicated to exhibit a significant difference $(P<0.05$, Table 4$)$.

\section{Histopathological examination of the rats' hippocampus}

As presented in Fig. 3, the hippocampus in the Control and DHA groups were normal (CA1) except for occasional vasodilatation, while the Model, Sev + DHA (0.3g/kg), Sev + DHA (1 g/ kg), Sev + DHA (3g/kg) groups all exhibited pathological change to some extent in the CA1 area (Fig. 2). Pathological changes, including the disordered arrangement of neurons, deep staining of neuronal nucleus pyknosis, cell edema and microglia foaming were observed in the Model group, and some areas were indicated to exhibit a small amount of cell necrosis. The pathological changes in the Sev + DHA (0.3g/kg), Sev + DHA (1 g/ kg), groups were slightly reduced compared with those in the Model group, and neurons exhibited a disordered arrangement, along with pyknosis and deep staining to neuronal nucleus, and cell edema was observed using a microscope. Pathological changes in the Sev + DHA $(3 \mathrm{~g} / \mathrm{kg})$ group did not change significantly.

\section{The changes of SOD, MDA and GSH-Px content}

As shown in Table 5, SOD and GSH-Px activity were significantly decreased in Model group $(P<0.01)$, compared with Control and DHA group rats. DHA treatment caused a significant increase in SOD and GSH-Px activity in Sev + DHA (0.3g/kg), Sev + DHA (1 $/ \mathrm{kg})$, Sev + DHA $(3 \mathrm{~g} / \mathrm{kg})$ groups $(P<0.05)$. However, there was no significant difference in SOD or GSH-Px activity between Control group and DHA group. The content of MDA in Control and DHA groups had no obvious difference. After repeated sevoflurane anesthesia, the content of MDA was significantly increased in Model group compared with Control and DHA groups $(P<0.05)$. DHA treatment caused a significant decrease in MDA activity in Sev + DHA $(0.3 \mathrm{~g} / \mathrm{kg})$, Sev + DHA $(1 \mathrm{~g} / \mathrm{kg})$, Sev + DHA $(3 \mathrm{~g} / \mathrm{kg})$ groups $(P<0.05)$. The results indicated that supplement with DHA may reduce the oxidative stress caused by repeated sevoflurane anesthesia.

\section{Changes in Nrf2 and HO-1 protein}

The results of immunohistochemistry demonstrated that the positive expression of Nrf2 and HO-1 was mainly located in the hippocampus and cortex of the brain. The positive expression in Control and DHA groups were not obvious. The positive expression in the Sev + DHA $(0.3 \mathrm{~g} / \mathrm{kg}), \operatorname{Sev}+\mathrm{DHA}(1 \mathrm{~g} / \mathrm{kg}), \operatorname{Sev}+$ DHA $(3 \mathrm{~g} / \mathrm{kg})$ groups were gradually increased. The Sev + DHA $(3 \mathrm{~g} / \mathrm{kg})$ group was significantly increased 
compared with the model group $(\mathrm{P}<0.01$; Fig. 4). The results were consistent with the results of western blot analysis.

Compared with the control and DHA groups, the expression of $\mathrm{Nrf} 2$ and $\mathrm{HO}-1$ protein in the model group was increased ( $P<0.05$; Fig. 5), and the expression of Nrf2 and HO-1 protein in the Sev + DHA (0.3g/kg), Sev + DHA (1 $\mathrm{g} / \mathrm{kg})$, Sev + DHA (3g/kg) groups were more obvious. However, in the Sev + DHA $(3 \mathrm{~g} / \mathrm{kg})$ group, the Nrf2 and HO-1 protein was significantly increased $(P<0.01)$. The results indicated that DHA may decrease the damage of repeated anesthesia on the brain tissue of rats, and this mechanism may be associated with Nrf2 and HO-1 protein levels.

\section{Discussion}

A study has confirmed that long-term exposure to sevoflurane can lead to neurodevelopmental disorders and impaired learning and memory in juvenile animals [12]. In addition, clinical studies in elderly patients have also indicated that exposure to sevoflurane is associated with postoperative cognitive function [14]. The neurotoxicity of sevoflurane is time-and dose-dependent [15]. A study hase demonstrated that exposure to sevoflurane in pregnant rats can lead to brain damage in newborns within two weeks of birth [16]. Furthermore, behavioral experiments in grown rats have also indicated that the learning and memory abilities of the sevoflurane exposed group were lower compared with the control group. In laboratory animals exposed to sevoflurane during the peak period of neurodevelopment, synaptic plasticity and long-term potentiation were affected, and learning and memory ability was decreased $[17,18]$. In the current study, after rats were exposed to repeated sevoflurane anesthesia, they were observed to exhibit aimless movement, no effective search of the platform, a longer space exploration period and decreased time taken to cross the platform, which was consistent with the aforementioned studies.

DHA, which is one of the substances that forms the normal structure of the brain and is easily obtained from deep-sea fish oil, is often processed as a health product or a nutritional supplement to be added to food. A study has indicated that the incidence of neurodegenerative diseases is lower in populations that adopt a mediterranean diet, which may be related to the high content of DHA and other substances during long-term intake [19]. In studies assessing elderly rodents, DHA and/or eicosapentaenoic acid supplements have been indicated to improve cognitive performance and induce protection against neuroinflammation and oxidative stress [20,21]. In the present study, after different doses of DHA were provided to aged rats with repeated sevoflurane anesthesia, the spatial exploration ability and navigation ability were attenuated in a dose-dependent manner, indicating that DHA could effectively intervene and treat the spatial learning and memory impairment induced by repeated sevoflurane anesthesia. The histopathological examination results of the hippocampus in rats also suggested that DHA could improve brain function, which was damaged by repeated sevoflurane anesthesia, and alleviated cognitive impairment. Moreover, with an increasing dose of DHA, the intervention effect was enhanced in a dosedependent manner. The results of the current study suggested that DHA had a protective effect on learning and memory impairment induced by repeated sevoflurane anesthesia in aged rats. 
The activation of Nrf2/HO-1 is believed to serve an important role in the development of brain injury [22]. The Nrf2/HO-1 pathway serves a crucial role in the body's anti-oxidative stress response, and Nrf2 nuclear translocation is the key to HO-1 activation [23]. In the current study, we detected the content of SOD, GSH$\mathrm{Px}$, and MDA in hippocampal tissues, the results indicated that DHA ameliorated the state of oxidative stress. Immunohistochemistry and western blot analysis were used to verify whether DHA induced antioxidative stress via the Nrf2/HO-1 pathway. The results demonstrated that the expression of Nrf2 and HO-1 proteins were significantly increased in each dose group of DHA compared with the model group, and this effect was dose-dependent.

\section{Conclusions}

In conclusion, the results of the present study indicated that DHA exhibited a protective effect on learning and memory impairment in aged rats, which was caused by repeated sevoflurane anesthesia. The increasing expression of $\mathrm{Nrf} 2$ and $\mathrm{HO}-1$ indicated that the mechanism may associated with the Nrf2/HO1 signaling pathway.

\section{Abbreviations}

DHA: docosahexaenoic acid

ELISA: enzyme linked immunosorbent assay

Nrf2: nuclear factor erythroid-2-related factor 2

HO-1: heme oxygenase 1

MDA: malondialdehyde

GSH-Px: glutathione peroxidase

BSA: bovine serum albumin

DAB: 3, 3-diaminobenzidine tetrahydrochloride

SDS-PAGE: polyacrylamide gel electrophoresis

SOD: superoxide dismutase

PVDF: polyvinylidene fluoride

\section{Declarations}

\section{Ethics approval and consent to participate}


The present study was approved by Ethics Committee of the Affiliated Yantai Yuhuangding Hospital of Qingdao University (YHDQD2055).

\section{Consent for publication}

Not applicable.

\section{Availability of data and materials}

The datasets generated and/or analyzed during the current study are available from the corresponding author on reasonable request.

\section{Competing interests}

There is no conflict of interest in this study.

\section{Funding}

This research was supported by Science and Technology Project of Yantai City (Project No.: 2016WS009). The funding provided experimental equipment and implementation for the study.

\section{Author's contributions}

TM, LK and ZX designed the study. TM and LK performed experiments. TM, LK and ZX performed data analysis, interpreted the data and acquired samples. TM and LK contributed pathological analysis. TM and ZX wrote the manuscript. All authors read and approved the final manuscript.

\section{Acknowledgments}

Not applicable.

\section{References}

1. Gross AF, Stern TA. Neuropsychiatric conditions associated withanesthesia exposure. Psychosomatics. 2014; 55(1): 21-28.

2. Yufune S, Satoh Y, Akai R, Yoshinaga Y, Kobayashi Y, Endo S, Kazama T. Suppression of ERK phosphor rylation through oxidative stress is involved in the mechanism under lying sevofluraneinduced toxicity in the developing brain. Sci Rep. 2016; 6: 21859.

3. Kabuto H, Amakawa M, Mankura M, Yamanushi TT, Mori A. Docosahexaenoic acid ethyl ester enhances 6-hydroxydopamine-induced neuronal damage by induction of lipid peroxidation in mouse striatum. Neurochem Res. 2009; 34(7): 1299-1303.

4. Hartmann T, van Wijk N, Wurtman RJ, Olde Rikkert MG, Sijben JW, Soininen $\mathrm{H}$, et al. A nutritional approach to ameliorate altered phospholipid metabolism in Alzheimer's disease. J Alzheimers Dis. 2014; 41(3): 715-717. 
5. Létondor A, Buaud B, Vaysse C, Fonseca L, Herrouin C, Servat B, Layé S, Pallet V, Alfos S. Erythrocyte DHA level as a biomarker of DHA status in specific brain regions of n-3 long-chain PUFAsupplemented aged rats. Br J Nutr. 2014; 112(11):1805-1818.

6. Dyall SC, Michael GJ, Whelpton R, Scott AG, Michael-Titus AT. Dietary enrichment with omega-3 polyunsaturated fatty acids reverses age-related decreases in the GluR2 and NR2B glutamate receptor subunits in rat forebrain. Neurobiol Aging. 2007;28(3):424-439.

7. Favrelière S, Perault MC, Huguet F, De Javel D, Bertrand N, Piriou A, Durand G. DHA-enriched phospholipid diets modulate age-related alterations in rat hippocampus. Neurobiol Aging. 2003;24(2):233-243.

8. Bang HY, Park SA, Saeidi S, Na HK, Surh YJ. Docosahexaenoic acid induces expression of heme oxygenase-1 and $\mathrm{NAD}(\mathrm{P}) \mathrm{H}$ : quinone oxidoreductase through activation of Nrf2 in human mammary epithelial cells. Molecules.2017;22(6):969.

9. Loboda A, Damulewicz M, Pyza E, Jozkowicz A, Dulak J. Role of Nrf2/HO-1 system in development, oxidative stress response and diseases: an evolutionarily conserved mechanism. Cellular and Molecular Life Sciences. 2016; 73(17):3221-3247.

10. Konrad FM, Knausberg U, Höne R, Ngamsri KC, Reutershan J. Tissue heme oxygenase-1 exerts antiinflammatory effects on LPS-induced pulmonary inflammation. Mucosal Immunology. 2015; 9(1): 98-111.

11. Wang LL, Yu QL, Han L, Ma XL, Song RD, Zhao SN, et al. Study on the effect of reactive oxygen species-mediated oxidative stress on the activation of mitochondrial apoptosis and the tenderness of yak meat. Food Chemistry. 2018; 244:394-402.

12. Piantadosi CA, Carraway MS, Babiker A, Suliman HB. Heme Oxygenase-1 Regulates Cardiac Mitochondrial Biogenesis via Nrf2-Mediated Transcriptional Control of Nuclear Respiratory Factor-1. Circulation Research. 2008; 103(11):1232-1240.

13. Cui Y, Ma S, Zhang C, Li D, Yang B, Lv P, et al. Pharmacological activation of the Nrf2 pathway by $3 \mathrm{H}-$ 1, 2-dithiole-3-thione is neuroprotective in a mouse model of Alzheimer disease. Behavioural Brain Research. 2018; 336:219-226.

14. Kilkenny C, Browne WJ, Cuthill IC, Emerson M, Altman DG. Improving bioscience research reporting: the ARRIVE guidelines for reporting animal research. PLoS Biol. 2010; 8: e1000412.

15. Qiu L, Zhu C, Bodogan T, Gómez-Galán M, Zhang Y, Zhou K, et al. Acute and Long-Term Effects of Brief Sevoflurane Anesthesia During the Early Postnatal Period in Rats. Toxicol Sci. 2016; 149(1): 121-133.

16. Fan D, Li J, Zheng B, Hua L, Zuo Z. Enriched Environment Attenuates Surgery-Induced Impairment of Learning, Memory, and Neurogenesis Possibly by Preserving BDNF Expression. Mol Neurobiol. 2016; 53(1): 344-354.

17. Van de Rest O, Wang Y, Barnes LL, Tangney C, Bennett DA, Morris MC. APOE epsilon4 and the associations of seafood and long-chain omega-3 fatty acids with cognitive decline. Neurology. 2016; 86(22): 2063-2070. 
18. Drobish JK, Gan ZS, Cornfeld AD, Eckenhoff MF. From the Cover: Volatile Anesthetics Transiently Disrupt Neuronal Development in Neonatal Rats. Toxicol Sci. 2016; 154(2): 309-319.

19. Zhao Y, Chen K, Shen X. Environmental Enrichment Attenuated Sevoflurane-Induced Neurotoxicity through the PPAR-gamma Signaling Pathway. Biomed Res Int. 2015; 2015: 107149.

20. Ho CF, Bon CP, Ng YK, Herr DR, Wu JS, Lin TN, et al. Expression of DHA-Metabolizing Enzyme Alox15 is Regulated by Selective Histone Acetylation in Neuroblastoma Cells. Neurochem Res. 2018; 43(3):540-555.

21. Valentini KJ, Pickens CA, Wiesinger JA, Tenton JI. The effect of fish oil supplementation on brain DHA and EPA content and fatty acid pro file in mice. Int J Food Sci Nutr. 2018; 69(6):705-717.

22. Chuang DY, Simonyi A, Kotzbauer PT, Gu Z, Sun GY. Cytosolic phospholi pase A2 plays a crucial role in ROS/NO signaling during microglialactivation through the lipoxygenase pathway. $\mathrm{J}$ Neuroinflamma tion. 2015; 12: 199.

23. Shu L, Wang C, Wang J, Zhang Y, Zhang X, Yang Y. The neuroprotection of hypoxic preconditioning on rat brain against traumatic brain injury by up-regulated transcription factor Nrf2 and HO-1 expression. Neuroscience Letters. 2016; 611:74-80.

24. Wang Z, Zhang H, Sun X, Ren L. The protective role of vitamin D3 in a murine model of asthma via the suppression of TGF- $\beta /$ Smad signaling and activation of the Nrf2/HO-1 pathway. Molecular Medicine Reports. 2016; 14(3):2389-2396.

\section{Tables}

\section{Tables}

Table 1 Changes of the rats body weight

\begin{tabular}{ccccc}
\hline Group & 1 day $(\mathrm{g})$ & 7 days $(\mathrm{g})$ & 14 days $(\mathrm{g})$ & 21 days $(\mathrm{g})$ \\
\hline Control & $584.52 \pm 21.16$ & $598.62 \pm 22.98$ & $613.62 \pm 21.08$ & $627.62 \pm 23.17$ \\
DHA $(3 \mathrm{~g} / \mathrm{kg})$ & $585.43 \pm 20.32$ & $601.17 \pm 20.34$ & $617.34 \pm 21.67$ & $633.19 \pm 24.58^{\#}$ \\
Model & $583.26 \pm 19.74$ & $597.13 \pm 20.69$ & $603.41 \pm 22.12$ & $608.41 \pm 23.36^{*}$ \\
Sev + DHA $(0.3 \mathrm{~g} / \mathrm{kg})$ & $582.31 \pm 19.42$ & $598.43 \pm 19.86$ & $606.51 \pm 20.82$ & $614.12 \pm 21.91^{\wedge}$ \\
Sev + DHA $(1 \mathrm{~g} / \mathrm{kg})$ & $584.65 \pm 20.97$ & $599.49 \pm 21.06$ & $608.27 \pm 21.94$ & $616.39 \pm 22.63$ \\
Sev + DHA $(3 \mathrm{~g} / \mathrm{kg})$ & $583.36 \pm 20.09$ & $599.78 \pm 21.43$ & $611.03 \pm 22.06$ & $619.56 \pm 23.74$
\end{tabular}

Compared with Control group, *P<0.05; compared with Model group, ${ }^{*} \mathrm{P}<0.05 \llbracket$ compared with DHA group, ${ }^{\wedge} \mathrm{P}<0.05$. 
Table 2 Changes of the rats heart rate (HR), respiratory frequency (RF) and blood oxygen saturation (BOS).

\begin{tabular}{|c|c|c|c|}
\hline Group & HR (beat/min) & RF (time/min) & BOS (\%) \\
\hline Control & $316.54 \pm 10.69$ & $92.34 \pm 5.12$ & $94.23 \pm 2.47$ \\
\hline DHA (3 g/kg) & $309.45 \pm 10.82^{\#}$ & $93.76 \pm 4.96^{\#}$ & $96.52 \pm 2.15^{\#}$ \\
\hline Model & $403.17 \pm 12.76^{*}$ & $78.52 \pm 6.07^{*}$ & $82.45 \pm 1.59^{*}$ \\
\hline $\mathrm{Sev}+\operatorname{DHA}(0.3 \mathrm{~g} / \mathrm{kg})$ & $383.26 \pm 11.89^{* \#^{\wedge}}$ & $86.34 \pm 5.76^{\# \wedge}$ & $84.06 \pm 1.67^{* \wedge}$ \\
\hline Sev + DHA $(1 \mathrm{~g} / \mathrm{kg})$ & $372.47 \pm 11.63^{* \#^{\wedge}}$ & $84.53 \pm 5.24^{\# \wedge}$ & $85.26 \pm 1.54^{* \wedge}$ \\
\hline $\mathrm{Sev}+\mathrm{DHA}(3 \mathrm{~g} / \mathrm{kg})$ & $374.12 \pm 11.26^{* \#^{\wedge}}$ & $85.01 \pm 5.45^{\# \wedge}$ & $87.19 \pm 1.73$ \\
\hline
\end{tabular}

Compared with Control group, ${ }^{*} \mathrm{P}<0.05$; compared with Model group, ${ }^{\#} \mathrm{P}<0.05 \llbracket$ compared with DHA group, ${ }^{\wedge} \mathrm{P}<0.05$.

Table 3 Escape latency period of the rats at different time points. $(n=9, \pm S)$ 


\begin{tabular}{|c|c|c|c|c|c|}
\hline Groups & Day 1 (sec) & Day 2 (sec) & Day 3 (sec) & Day 4 (sec) & Day 5 (sec) \\
\hline $\mathrm{Cc}$ & 7 & 47.68 & 38.81 & 30.61 & 19.5 \\
\hline DHA & $57.93 \pm 3.92^{\#}$ & $47.12 \pm 3.54^{\#}$ & $37.98 \pm 3.21^{\#}$ & $29.94 \pm 2.71^{\#}$ & $18.69 \pm 1.73^{\#}$ \\
\hline Model & $82.29 \pm$ & $70.56 \pm$ & $59.39 \pm$ & 43.89 & 38.56 \\
\hline $\mathrm{Sev}+\mathrm{DHA}$ & $75.69=$ & $67.01=$ & 53.18 & 40.4 & 32.7 \\
\hline $\mathrm{Sev}+\mathrm{DHA}(1 \mathrm{~g} / \mathrm{kg})$ & $69.19 \pm 2.22^{*}$ & $58.84 \pm 3$ & 48.98 & 34.5 & 26.85 \\
\hline Sev + DHA (3 g/kg) & $61.57 \pm 2.78^{\#}$ & $53.22 \pm 1.91^{\#}$ & $40.62 \pm 1.72^{\#}$ & $32.41 \pm 2.21^{\#}$ & $20.01 \pm 2.07^{\#}$ \\
\hline
\end{tabular}

Compared with Control group, ${ }^{*} \mathrm{P}<0.05$; compared with Model group, ${ }^{*} \mathrm{P}<0.05 \square$ compared with DHA group, ${ }^{\wedge} \mathrm{P}<0.05$.

Table 4 Comparison of learning ability test results in each group $(n=9, \pm S)$

\begin{tabular}{ccc}
\hline Groups & Number of Times to Cross the Platform & Time for Target Quadrant Stay (sec) \\
\hline Control & $8.63 \pm 1.07$ & $67.68 \pm 13.69$ \\
DHA $(3 \mathrm{~g} / \mathrm{kg})$ & $8.81 \pm 0.98^{\#}$ & $68.49 \pm 13.54^{\#}$ \\
Model & $4.96 \pm 0.63^{*}$ & $42.56 \pm 9.31^{*}$ \\
Sev + DHA $(0.3 \mathrm{~g} / \mathrm{kg})$ & $6.63 \pm 0.79^{* \wedge}$ & $57.08 \pm 11.12^{* \wedge}$ \\
Sev + DHA $(1 \mathrm{~g} / \mathrm{kg})$ & $7.29 \pm 0.52$ & $60.84 \pm 13.54^{\#}$ \\
Sev + DHA $(3 \mathrm{~g} / \mathrm{kg})$ & $8.17 \pm 0.38^{\#}$ & $63.97 \pm 11.91^{\#}$ \\
\hline
\end{tabular}


Compared with Control group, ${ }^{*} \mathrm{P}<0.05$; compared with Model group, ${ }^{*} \mathrm{P}<0.05 \llbracket$ compared with DHA group, ${ }^{\wedge} \mathrm{P}<0.05$.

Table 5 Detection of oxidative damage index on rats brain

\begin{tabular}{|c|c|c|c|}
\hline Group & $\mathrm{SOD}(\mathrm{U} / \mathrm{mgP})$ & $\mathrm{MDA}(\mathrm{nmol} / \mathrm{mgP})$ & GSH-Px (U/L) \\
\hline Control & 9 & .52 & 15.5 \\
\hline DHA (3 g/kg) & $3.74 \pm 0.62^{\#}$ & $2.36 \pm 0.42^{\#}$ & $15.61 \pm 2.11^{\#}$ \\
\hline Model & $2.17 \pm 0.36^{*}$ & $5.02 \pm 0.87^{*}$ & $8.45 \pm 1.42^{*}$ \\
\hline $\mathrm{Sev}+\mathrm{DHA}(0.3 \mathrm{~g} / \mathrm{kg})$ & $2.26 \pm 0.41^{* \#^{\wedge}}$ & $4.34 \pm 0.81^{* \#^{\wedge}}$ & $9.68 \pm 1.24^{* \wedge}$ \\
\hline Sev + DHA $(1 \mathrm{~g} / \mathrm{kg})$ & $2.78 \pm 0.42^{* \# \wedge}$ & $3.63 \pm 0.73^{* \#^{\wedge}}$ & $11.26 \pm 1.43^{* \wedge}$ \\
\hline Sev + DHA (3 g/kg) & $3.26 \pm 0.47^{\#^{\wedge}}$ & $2.81 \pm 0.56^{\#^{\wedge}}$ & $13.89 \pm 1.96^{\# \wedge}$ \\
\hline
\end{tabular}

Compared with Control group, ${ }^{*} \mathrm{P}<0.05$; compared with Model group, ${ }^{*} \mathrm{P}<0.05 \llbracket$ compared with DHA group, ${ }^{\wedge} \mathrm{P}<0.05$. 


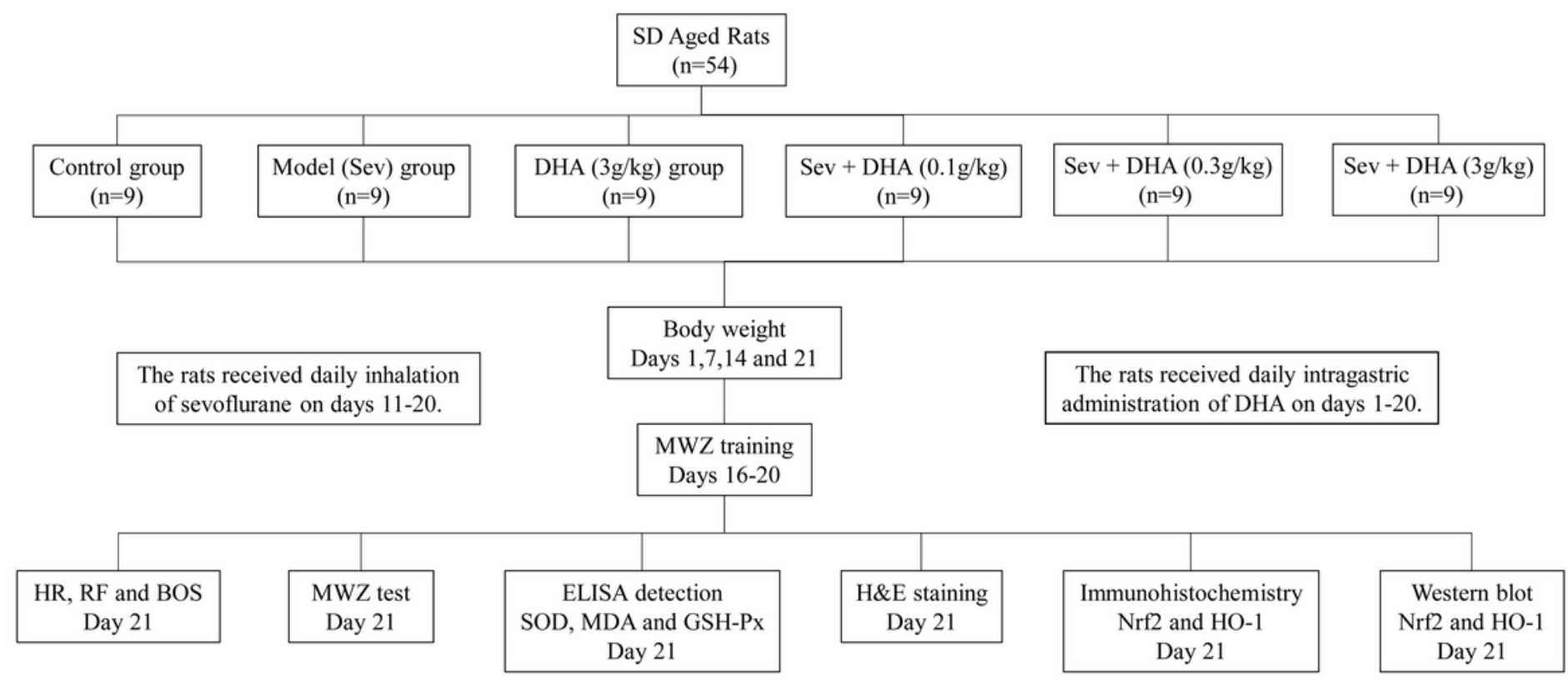

\section{Figure 1}

The experimental flow chart.

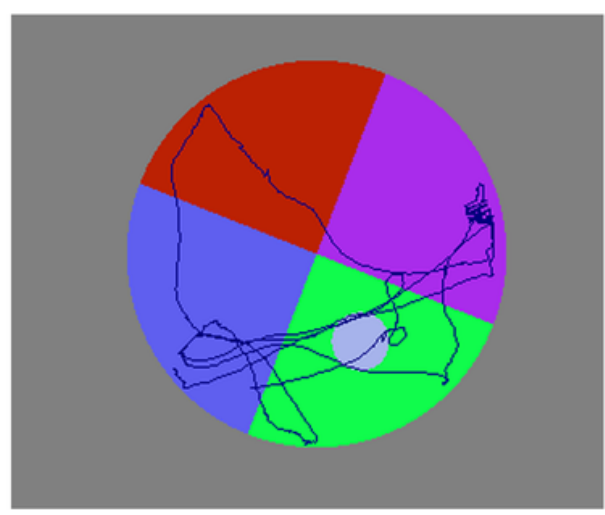

Control

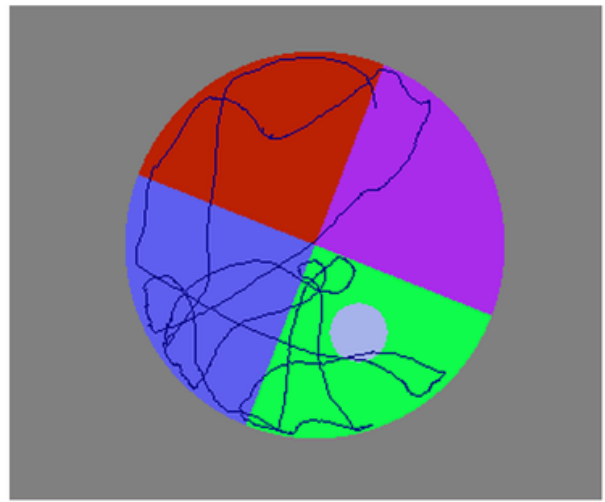

Sev + DHA $(0.3 \mathrm{~g} / \mathrm{kg})$

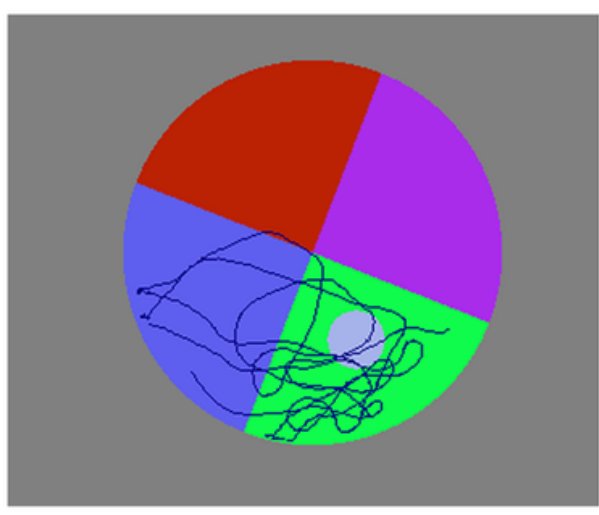

DHA (3 g/kg)

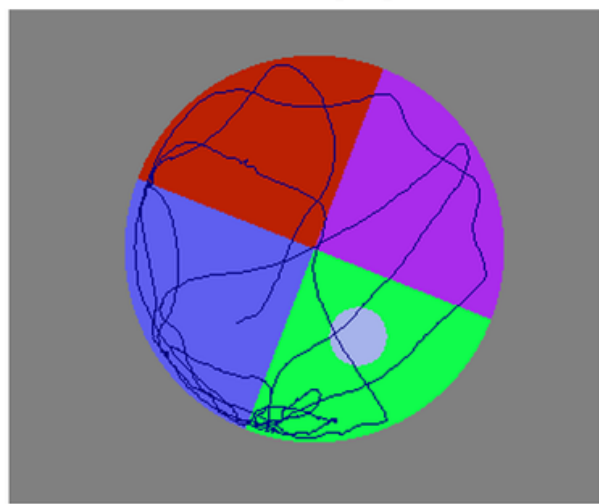

Sev + DHA $(1 \mathrm{~g} / \mathrm{kg})$

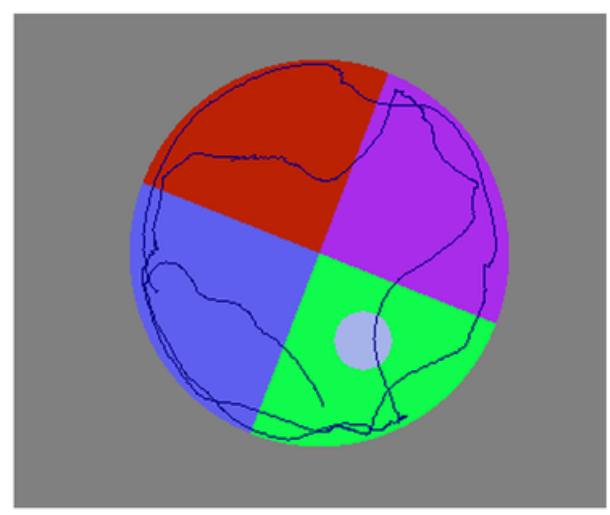

Model

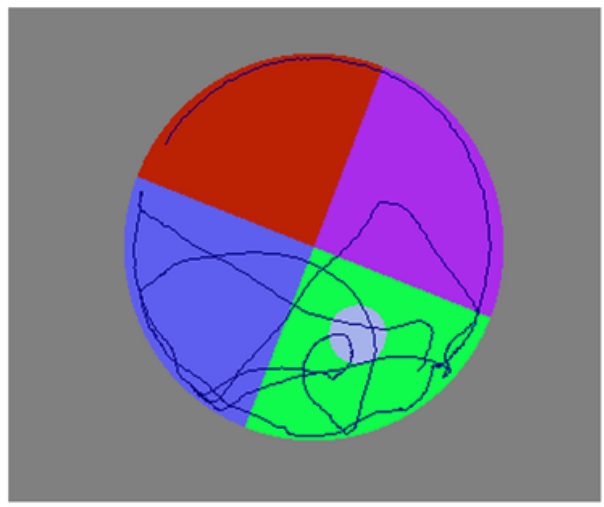

Sev + DHA (3 g/kg)

\section{Figure 2}

The swimming trajectory of each group. 


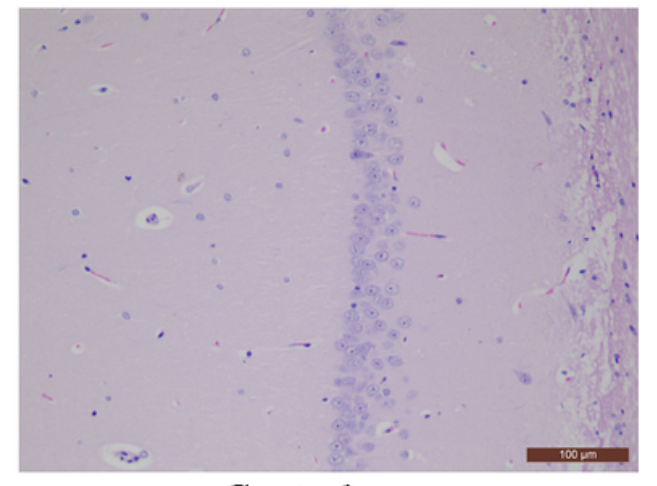

\section{Control}

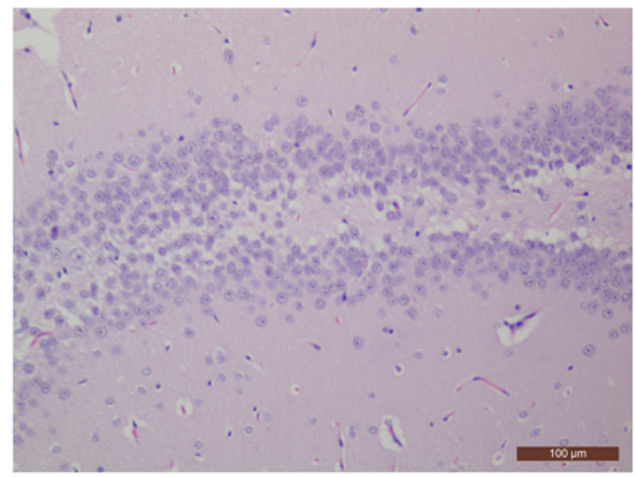

Sev + DHA $(0.3 \mathrm{~g} / \mathrm{kg})$

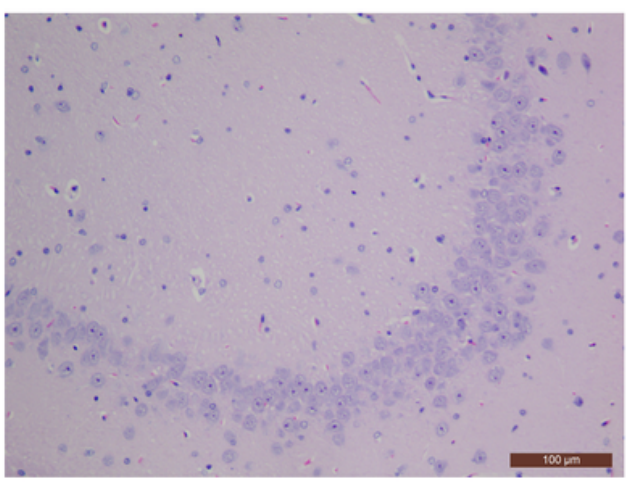

DHA $(3 \mathrm{~g} / \mathrm{kg})$

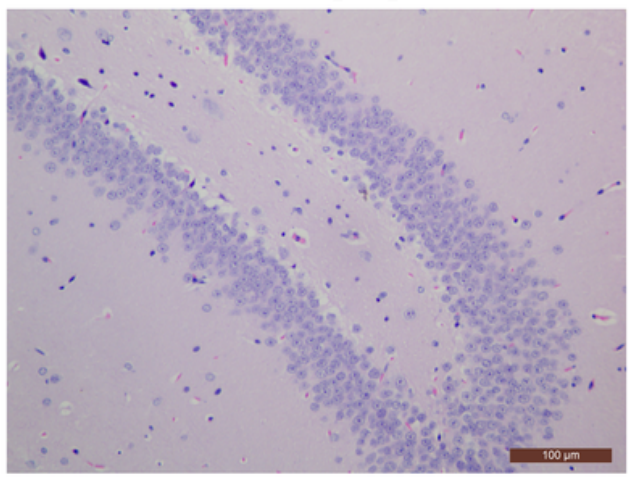

Sev + DHA $(1 \mathrm{~g} / \mathrm{kg})$

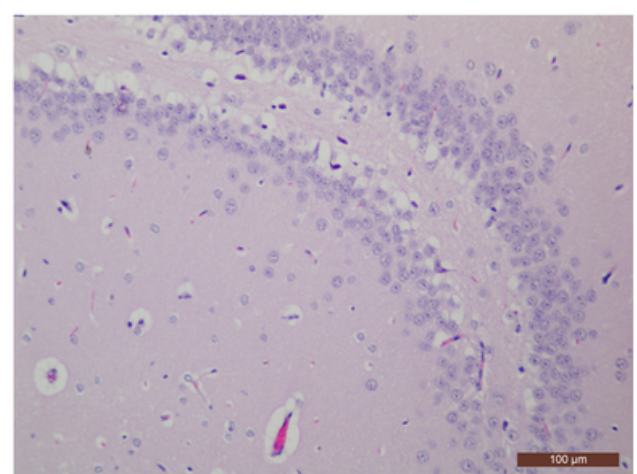

Model

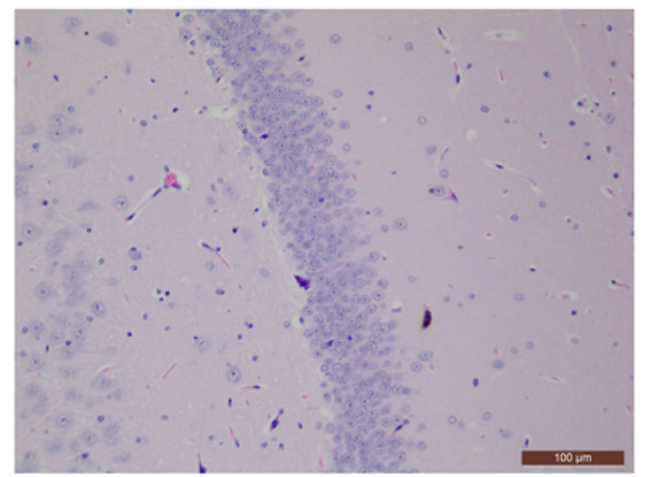

$\mathrm{Sev}+\mathrm{DHA}(3 \mathrm{~g} / \mathrm{kg})$

\section{Figure 3}

The pathological changes in the hippocampus measured using haematoxylin and eosin staining. The magnification was $\times 200$; scale bar, $100 \mu \mathrm{m}$. 

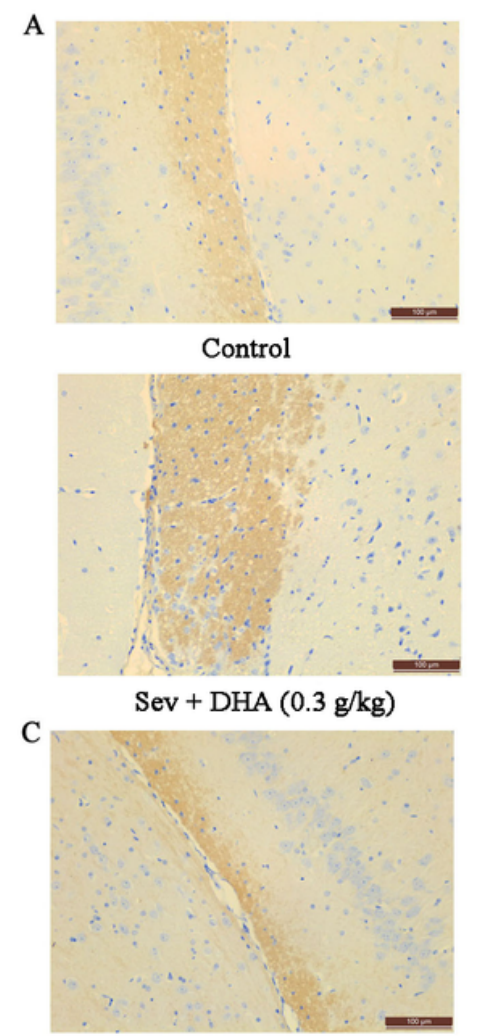

Control

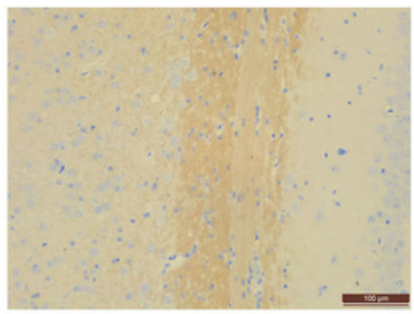

$\mathrm{Sev}+\mathrm{DHA}(0.3 \mathrm{~g} / \mathrm{kg})$

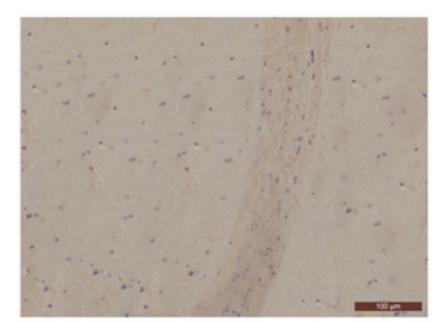

DHA (3 g/kg)

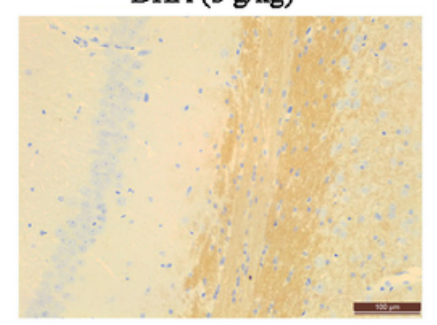

Sev + DHA $(1 \mathrm{~g} / \mathrm{kg})$

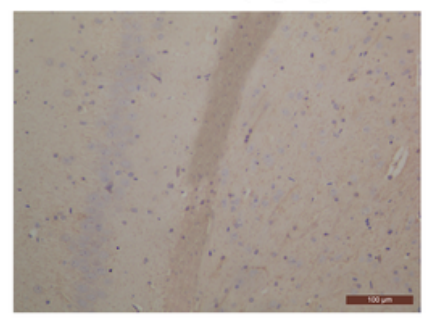

$\mathrm{DHA}(3 \mathrm{~g} / \mathrm{kg})$

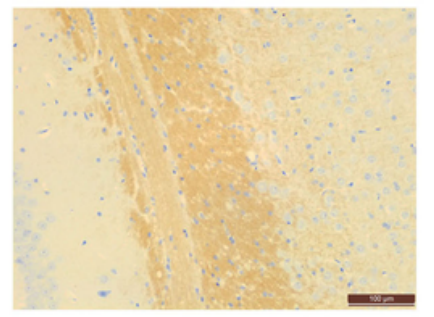

$\mathrm{Sev}+\mathrm{DHA}(1 \mathrm{~g} / \mathrm{kg})$

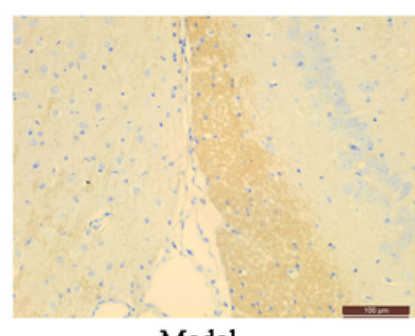

Model

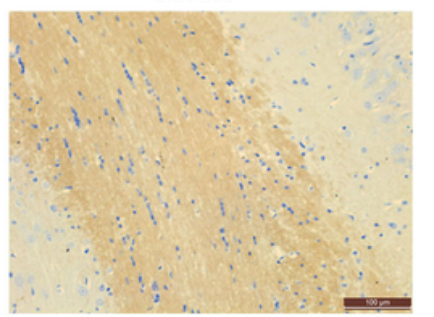

$\mathrm{Sev}+\mathrm{DHA}(3 \mathrm{~g} / \mathrm{kg})$

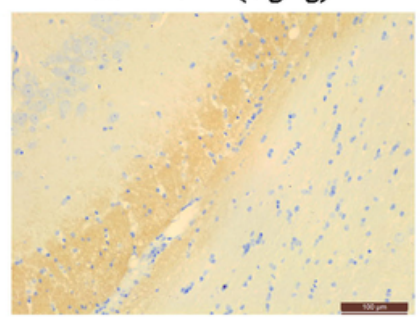

Model

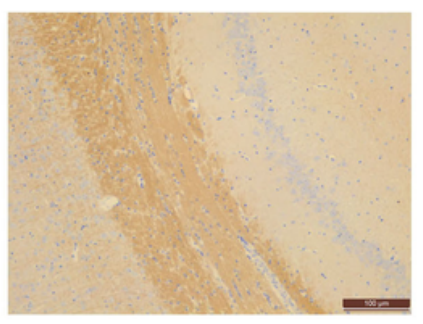

Sev + DHA $(3 \mathrm{~g} / \mathrm{kg})$
B

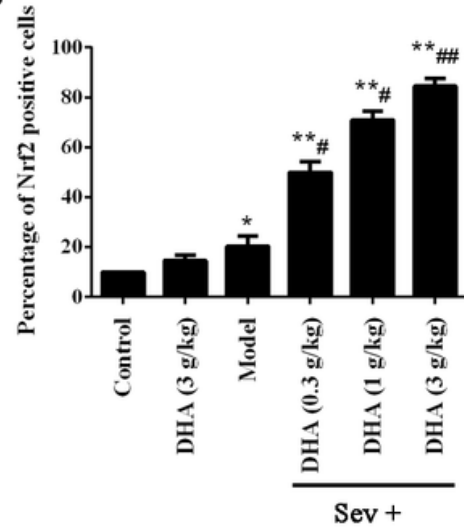

D

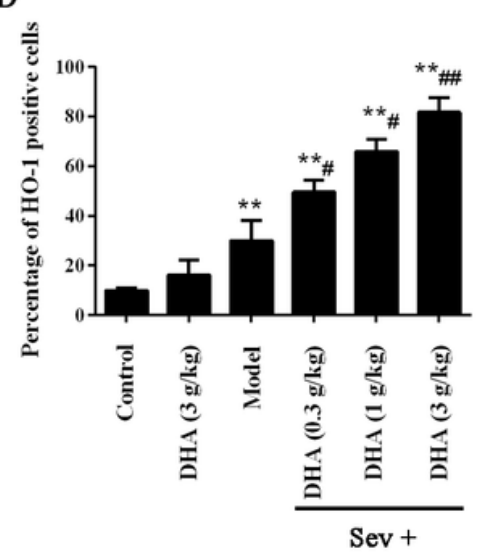

Figure 4

The expression of Nrf2 and HO-1 detected by immunohistochemistry. The magnification was x200; scale bar, $100 \mu \mathrm{m}$. (A) The expression of Nrf2 protein. (B) Percentage of Nrf2 positive cells from each group. (C) The expression of HO-1 protein. (D) Percentage of HO-1 positive cells from each group. ${ }^{*} \mathrm{P}<0.05$ vs. Control or DHA ( $3 \mathrm{~g} / \mathrm{kg})$ group, ${ }^{\star *} \mathrm{P}<0.01$ vs. Control group or DHA $(3 \mathrm{~g} / \mathrm{kg}) ; \# \mathrm{P}<0.05$ vs. Model group, $\# \# \mathrm{P}<0.01$ vs. Model group. 
A

Nrf2

HO-1

$\beta$-actin
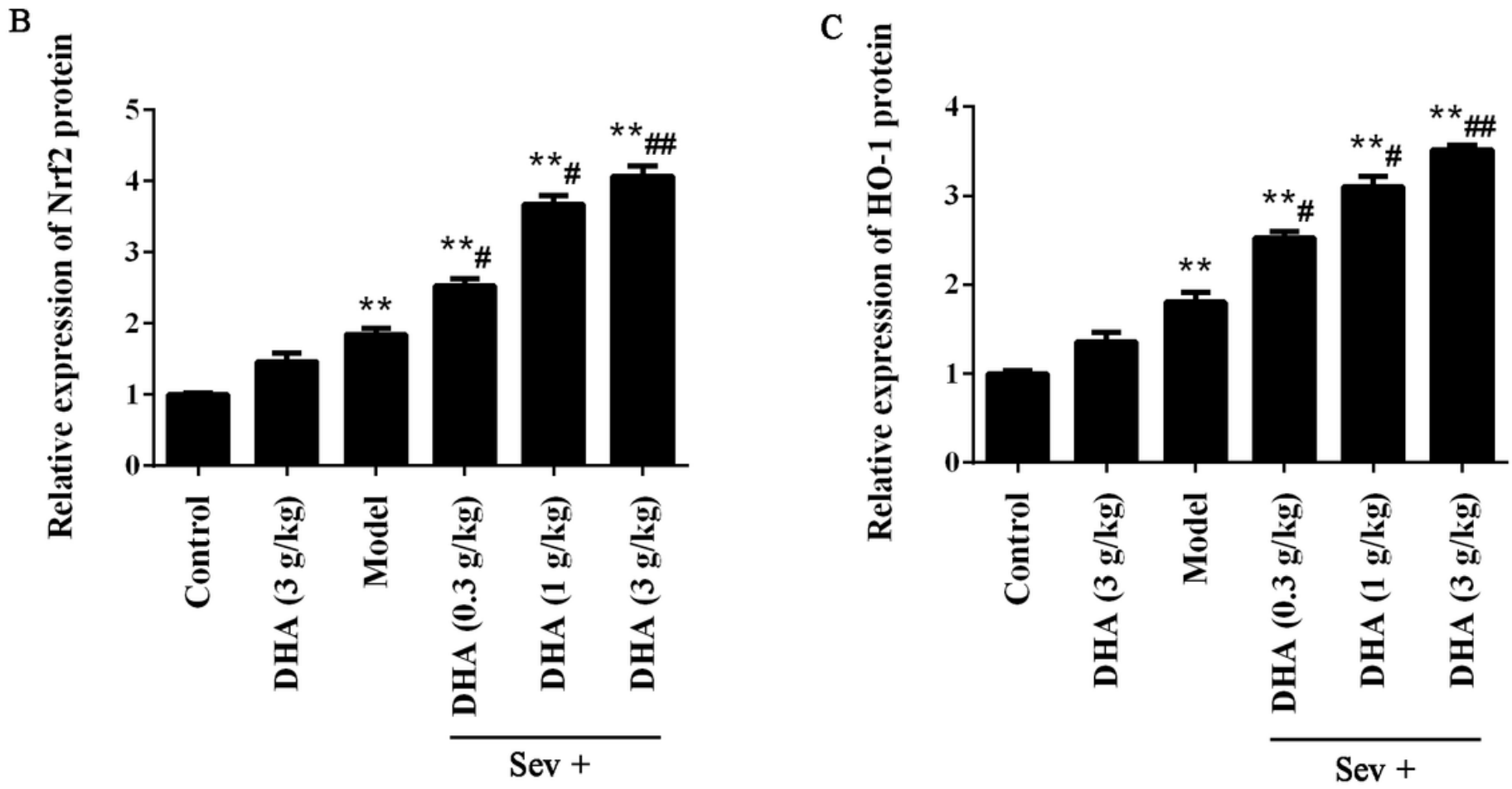

Figure 5

Nrf2 and HO-1 expression. (A) Western blot analysis was performed to determine the Nrf2 and HO-1 protein level in each group. (B) Quantification of the Nrf2 protein expression. (C) Quantification of the HO1 protein expression. ${ }^{\star} \mathrm{P}<0.05$ vs. Control group or DHA $(3 \mathrm{~g} / \mathrm{kg}),{ }^{\star} \mathrm{P}<0.01$ vs. Control group or DHA (3 $\mathrm{g} / \mathrm{kg})$; \#P<0.05 vs. Model group, \#\#P<0.01 vs. Model group.

\section{Supplementary Files}

This is a list of supplementary files associated with this preprint. Click to download.

- Nrf2.bmp

- H01.bmp

- renameda5bcb.bmp 
- NC3RsARRIVEGuidelinesChecklist2014.pdf 